\title{
Dermatotoxicology of microneedles (MNs) in man
}

\author{
John Havens Cary ${ }^{1}$ (D) $\cdot$ Becky S. Li ${ }^{2} \cdot$ Howard I. Maibach ${ }^{3}$
}

Published online: 4 July 2019

(C) Springer Science+Business Media, LLC, part of Springer Nature 2019

\begin{abstract}
Developed within the last few decades, microneedles (MNs) have only recently seen wide-scale use among the general population, especially in the area of cosmetics. With the FDA only starting to regulate microneedling devices and the many new microneedling products that enter the modern global market, it is of utmost importance to establish the safety profile and reasonable expectations of the microneedling practice and its products. In our review of current literature, the authors searched the keyword "microneedle" with the following terms: "safety", "side effect", "toxicology", "adverse effect", "adverse event", "infection", "dermatitis", "granuloma", "scarring", and "hyperpigmentation". Despite wide-scale implementation of MNs, we are likely only beginning to understand the potential of MNs as a medical and consumer product, and we should, therefore, be aware of any potential adverse events associated with the product.
\end{abstract}

Keywords Microneedles $\cdot$ Dermatotoxicology $\cdot$ Hyperpigmentation $\cdot$ Infection $\cdot$ Contact dermatitis $\cdot$ Skin

\section{Introduction}

Microneedles (MNs), minimally invasive devices designed to painlessly penetrate stratum corneum, were developed in 1976 as a means for more efficient transdermal drug delivery (Ma and $\mathrm{Wu}$ 2017). In subsequent decades, advancement in MN technology and manufacturing has led to development of several types of MNs including: hollow, solid, dissolving, coated, and hydrogel forming (Nguyen and Park 2018). Hollow MNs deliver drugs through a channel in a similar manner to hypodermic needles, while solid MNs are more frequently used in pretreatment to enhance the permeability of the skin before

John Havens Cary

John.Cary@ucsf.edu; havenscary@gmail.com

$\triangle$ Becky S. Li

Becky.Li@ucsf.edu; beckysiyunli@gmail.com

Howard I. Maibach

Howard.Maibach@ucsf.edu

1 Louisiana State University School of Medicine, 433 Bolivar Street, New Orleans, LA 70112, USA

2 Howard University College of Medicine, $520 \mathrm{~W}$ Street NW, Washington, DC 20059, USA

3 School of Medicine, Department of Dermatology, University of California, San Francisco, 90 Medical Center Way, Box 0989, Surge Building, Room 110, San Francisco, CA 94143-0989, USA application of a topical product. Dissolving MNs are constructed from a biodegradable polymer or polysaccharide with therapeutic molecules contained within; coated MNs contain the drug formulation on the outside surface of the needles. Lastly, hydrogel-forming MNs are made of expanding material with an active agent attached to the baseplate (Nguyen and Park 2018). The following introduction briefly states some of the promising potential applications of MNs, including dermal and intrascleral drug delivery, vaccine administration, blood and interstitial fluid extraction, as well as numerous uses in cosmetics (Ramaut et al. 2018; Ma and Wu 2017).

MNs allow delivery of higher molecular weight and hydrophilic drugs that would otherwise be unable to diffuse across the stratum corneum. In contrast to enteral drug delivery, dermal drug delivery avoids stomach degradation and hepatic first pass metabolism, while it may also produce higher drug concentrations in the dermis and other target tissues. MN transdermal drug delivery is a continually growing field with increasing patents filed every year for numerous drugs. Here, we briefly discuss just a few of the many studies involving MN drug delivery. Badran et al. (2009) demonstrated effective penetration of radiolabeled mannitol, a hydrophilic drug expected to have poor penetration into the stratum corneum, in full thickness human skin grafts when combined with microneedling. Dhurat et al. found enhanced effectiveness of minoxidil in men with androgenetic alopecia when used in conjunction with micronneedling in comparison to conventional minoxidil treatment; they also 
demonstrated effectiveness of MN and minoxidil combination therapy in patients who failed to respond to conventional minoxidil treatment (Dhurat et al. 2013; Dhurat and Mathapati 2015). Jiang et al. (2009) demonstrated effective intrascleral delivery of microparticles via MNs. With possible drug diffusion to surrounding tissues like the choroid, retina, and ciliary body, intrascleral drug delivery via MNs has the potential to treat posterior eye disease such as glaucoma and macular degeneration although further research is needed (Jiang et al. 2009).

While most MN vaccination studies have focused on delivery of influenza antigen, many other applications exist for $M N$ vaccines. MN delivery of vaccines releases antigenic material into the viable dermis, which has shown stronger immunological reactions when compared to muscle, the delivery target of traditional hypodermic needles (Engelke et al. 2015). A multi-center, randomized open-label study in 978 healthy adults showed intradermal influenza vaccine delivered by microinjection systems to convey a non-inferior humoral response against three influenza strains and two superior humoral responses to both A strains (H1N1, H3N2) (LerouxRoels et al. 2008). Similar studies on influenza vaccination have demonstrated comparable or superior responses when compared to responses from conventional intramuscular vaccination (Van Damme et al. 2009; Kenney et al. 2004). Other studies have demonstrated effective levels of neutralizing antibodies in $100 \%$ of measles vaccinated non-human primates compared to $75 \%$ of subcutaneously vaccinated non-human primates (Joyce et al. 2018). In a phase I, partly blinded controlled trial, authors found no significant statistical difference in antibody titers among groups vaccinated with healthcare-administered intramuscular hypodermic needle influenza vaccine, healthcare-administered MN influenza vaccination patch, and self-administered MN influenzavaccination patch, suggesting a possible future for selfadministered vaccines (Rouphael et al. 2017). Chiefly, MNs have application in global health vaccination due to ability for non-climate controlled distribution and potential for self-administration. In addition, MNs eliminate sharp, bio-hazardous waste, painful injections, and potential needle-stick injuries present in vaccines administered via hypodermic needles.

Development of optimized hollow MNs has resulted in additional potential applications, including blood and interstitial fluid extraction (Li et al. 2013; Kiang et al. 2017). When paired with biosensors and other microsystems, MNs have the potential for use in glucose and drug monitoring (Kiang et al. 2017). While experiments with MN-integrated biosensors have yielded promising results, extensive pre-clinical and clinical trials are needed before their implementation as point-ofcare devices for ISF collection, drug concentration assessment, or dosing in real time (Kiang et al. 2017).

Dermal microneedling has proven an effective therapy for atrophic acne scars and skin rejuvenation (Kim et al. 2011).
Although the exact mechanism is unknown, MN puncture likely disrupts older collagen strands and promotes damaged collagen removal (Fabbrocini et al. 2009). In addition, rolling with multiple MNs to promote new collagen and elastin deposition, known as collagen induction therapy, has become popular practice in treating disfiguring scars and rhytides and rejuvenating skin (Fabbrocini et al. 2009; Aust et al. 2008). Other methods used to improve the clinical appearance of acne scarring such as laser resurfacing pose potential risks associated with a disrupted skin epidermal barrier. Cho et al. (2012) showed microneedling to lead to improved grade of acne scars and global assessment of large pores in more than $70 \%$ of thirty tested patients. Kim et al. (2011) compared the effects of microneedling versus intense pulsed light therapy (IPL) for skin rejuvenation, finding significantly higher levels of collagen in microneedling when evaluated via caliper, microscopic examination, Western blot analysis for type I collagen, and enzyme-linked immunosorbent assay (ELISA) for total collagen content.

\section{Adverse events and prevention}

Perhaps the most extensive use of MNs in any one application occurs with collagen-induction therapy. Consequently, most discussion of adverse events and normal post-procedural expectations involve this application.

Common contraindications to $\mathrm{MN}$ collagen-induction therapy include: active acne; herpes labialis or other active current infection such as impetigo or warts; patients on anticoagulant therapy such as warfarin and heparin or with other blood dyscrasias; patients with extreme keloid scarring tendency; and patients on chemotherapy, radiotherapy, or high doses of corticosteroids (Singh and Yadav 2016; Fernandes 2005).

Prior to MN collagen-induction therapy, patients should use a cleanser to remove any makeup or debris prior to application of any topical aesthetic cream/gel to treatment area (Alster and Graham 2018). Alster and Graham (2018) typically apply a compounded $30 \%$ lidocaine cream for 20-30 min before removal with water-soaked gauze and alcohol prep immediately before therapy. In the healthy patient without contraindication to treatment, typical post-procedural expectations in collagen induction therapy with MNs include: pinpoint bleeding, bruising, erythema, and irritation (Fernandes 2005). Fernandes (2005) outlines a predicted appearance timeline and general recommendations following percutaneous collagen induction. Patients may note a bruised skin appearance with a swollen face following collagen induction therapy with a minimal, temporary ooze of serum. He recommends soaking the skin with saline swabs for $1-2 \mathrm{~h}$ followed by treatment with a Tea-tree oil cleanser, while also encouraging patients to use topical vitamin A and vitamin C cream or oil to enhance healing. Other authors recommend use of hyaluronic 
acid gel in the first $4 \mathrm{~h}$ post-treatment followed by $1 \%$ hydrocortisone cream or nonallergenic moisturizing cream after $4 \mathrm{~h}$ (Alster and Graham 2018). However, it is important that patients use only topical products that are approved for intradermal use, as inappropriate topical formula in conjunction with $\mathrm{MN}$ therapy increases the potential for allergic and irritant reactions. Note that vitamin A is known to aggravate flush and cause dry, flaky skin. Patients should thoroughly wash his/her face until all serum, blood, and oil is removed to prevent any minor eschar formation, as minor eschars may result in development of simple milia or tiny pustules. The skin should look less dramatic following day 1 with moderate flush on day 4 to 5 and very few visible signs post-procedure day 7 . Patients should avoid unapproved topical formulations for at least $24 \mathrm{~h}$ and refrain from direct sun exposure for at least 10 days. Fernandes advises at least one month in between retreatment with MNs, while Alster and Graham (2018) recommend biweekly intervals until desired outcome is achieved.

Despite the potential uses and existence for over 4 decades, MNs are yet to be fully integrated into the medical system. The following discussion investigates potential side effects of MNs, including: infection, irritation and irritant contact dermatitis, allergic contact dermatitis, hyperpigmentation, abnormal scarring, and irritant and allergic granulomas. We also consider the potential for photoirritation/phototoxicity, photoallergic contact dermatitis, non-immunologic contact urticaria, and immunologic contact urticaria. For a summary of literature documenting adverse events with MNs, please see Table 1.

\subsection{Infection}

The stratum corneum serves many purposes, including a physical defense against microorganisms. Microchannels formed during $\mathrm{MN}$ therapy has the potential to facilitate access of microorganisms and increase susceptibility to infection. Gupta et al. (2011) evaluated the kinetics of stratum corneum resealing in $\mathrm{MN}$ versus hypodermic needles, finding faster resealing in $\mathrm{MN}$-treated skin. Several others have evaluated skin resealing kinetics with MNs with studies finding closures times as quick as $15 \mathrm{~min}$ (Bal et al. 2010) with other studies finding closure within 2 (Gupta et al. 2011), 8-24 (Haq et al. 2009), and $24 \mathrm{~h}$ (Kelchen et al. 2016). Donnelly et al. (2009) evaluated the microbial penetration in hypodermic needles versus MN-induced holes in Silescol ${ }^{\circledR}$ membranes and neonate porcine skin. They demonstrated significantly lower penetration of Candida albicans, Pseudomonas aeruguinosa, and Staphylococcus epidermidis across viable epidermis in MN versus hypodermic needle puncture. Vicente-Perez et al. (2017) evaluated the effect of MN patches on mice, testing for biomarkers of inflammation and infection, weight, and several other variables. They found no detectable levels of TNF- $\alpha$ among mice and no statistically significant increase in C-reactive protein, immunoglobulin G, or interleukin 1-

Table 1 Literature documenting adverse events to microneedles (MNs)

\begin{tabular}{|c|c|c|c|}
\hline Adverse Reaction Type & Author(s) and Year & $\begin{array}{l}\text { Number } \\
\text { of patients }\end{array}$ & Additional details \\
\hline \multirow[t]{3}{*}{ Infection } & Aust et al. (2008) & 2 & 2/480 patients developed Herpes Simplex infection \\
\hline & Torezan et al. (2013) & 1 & $1 / 10$ patients developed infection based on symptoms \\
\hline & Cunha et al. (2017) & 1 & Microsporum canis infection of bilateral arms and legs \\
\hline Irritant Contact Dermatitis (ICD) & $\begin{array}{l}\text { Cercal Fucci-da-Costa and } \\
\text { Reich Camasmie (2018) }\end{array}$ & 1 & Suspected irritation to arnica-based cream \\
\hline $\begin{array}{l}\text { ACD Allergic Contact } \\
\text { Dermatitis (ACD) }\end{array}$ & Yadav and Dogra (2016) & 1 & $\begin{array}{l}\text { "rail track appearance" of adverse reaction with positive } \\
\text { patch test to nickel }\end{array}$ \\
\hline Allergic/Irritant Granulomas ${ }^{1}$ & Soltani-Arabshahi et al. (2014) & 3 & $\begin{array}{l}2 \text { patients with granulomatous reaction, systemic symptoms, } \\
\text { and }+1 \text { reaction to Vita C serum; } 1 \text { patient with granulomatous } \\
\text { reaction and no systemic symptoms }\end{array}$ \\
\hline \multirow[t]{2}{*}{ Irregular scarring } & Pahwa et al. $(2012)^{2}$ & 1 & $\begin{array}{l}\text { "tram tracking" appearance over temporal area, zygomatic arch, } \\
\text { and forehead }\end{array}$ \\
\hline & Dogra et al. $(2014)^{2}$ & 2 & $\begin{array}{l}\text { 2/36 patients in study presented with a "tram trek" adverse event; } \\
1 / 2 \text { "tram trek" patients withdrawn from study }\end{array}$ \\
\hline \multirow[t]{3}{*}{$\begin{array}{l}\text { Post-inflammatory } \\
\text { hyperpigmentation }\end{array}$} & Dogra et al. (2014) & 5 & $\begin{array}{l}5 / 36 \text { patients in study; } 3 / 5 \text { patients forced to withdraw from study; } \\
2 / 5 \text { patients improved with photoprotection }\end{array}$ \\
\hline & Sharad (2011) & 4 & $4 / 36$ patients in study with more transient hyperpigmentation \\
\hline & Majid (2009) & 1 & $1 / 37$ patients in study with transient hyperpigmentation \\
\hline
\end{tabular}

${ }^{1}$ Allergic/Irritant Granulomas may be considered a subset of ICD or ACD

2 "Tram tracking" or "tram trek" lesions may be due to ICD or ACD, as the authors did not determine the underlying cause 
beta in MN-tested mice in comparison to control mice, regardless of formulation type, needle density, number of applications, or mouse gender ( $p>0.05$ in all cases). In addition, mice in all study and control groups demonstrated increased weight over the course of the study. Collectively, the authors were unable to detect any infection or any variable indicative of an infection across all tested mice.

Aust et al. (2008) performed a retrospective analysis of 480 patients with fine wrinkles, lax skin, scarring, and striae gravidarum treated with percutaneous collagen induction using the Medical Roll-CIT with topical Vitamin A and C cosmetic creams for a minimum of 4 weeks post-operatively. Two patients developed herpes simplex infection following a full-face needling that was successfully treated with acyclovir.

In a pilot split-face study comparing conventional methyl aminolevulinate-photodynamic therapy (PDT) with microneedling-assisted PDT on actinically damaged skin, 1 of 10 patients developed an infection on the MN treated side 7 days post-treatment, determined by signs and symptoms of high local temperature, redness, pain, and crusts; however, the patient responded well to cephalosporin treatment (Torezan et al. 2013).

Cunha et al. (2017) document a case of tinea corporis that emerged in corresponding locations of dermaroller (540 stainless steel microneedles of $0.5 \mathrm{~mm}$ length) use approximately 3 weeks after start of her home MN therapy for bilateral scarring of her arms and legs. Potassium hydroxide examination of the lesions was positive for fungus with lesion culture growing Microsporum canis. While the patient confirmed skin-cleaning prior and sanitation of the MN device before and after therapy, inadequate sterilization of the patient's skin and $\mathrm{MN}$ device cannot be excluded. The patient experienced complete resolution after treatment with oral terbinafine and topical sertoconazol at 5-week follow up.

Leatham et al. (2018) document a case of facial autoinoculation of varicella via a home microneedling roller device. A healthy woman with a distant history of primary varicella zoster virus (VZV) infection and no prior shingles vaccination reported grouped lesions over her chest, which she presumed an acneiform eruption. The patient self-treated the area with an at-home microneedling roller device and used the device over her face to reduce the appearance of rhytides. On presentation, the patient had "grouped, eroded papules and vesicles on the right T4 dermatome" and "eroded papules on the forehead, lateral cheeks, many located at spaced distances." PCR yielded positive results for VZV. Patient was started on oral valacyclovir and was free of lesions and experienced no postherpetic neuralgia at 6 weeks.

\subsection{Irritant contact dermatitis}

Irritant contact dermatitis (ICD) is an eczematous-like reaction occurring from contact with a chemical, biologic, or physical agent (Tan et al. 2014). Severity of the reaction is dependent upon the physicochemical property of the agent and the degree of activation of the innate immune system (Tan et al. 2014). Keratinocytes, which comprise $95 \%$ of epidermal cells, are responsible for production of the majority of cytokines likely responsible for the ensuing erythema and edema (Tan et al. 2014). In contact with any agent, there is potential for ICD; however, it is likely that disruption of the stratum corneum provides increased susceptibility to a particular irritant. MNs are most frequently manufactured from nonirritating metal, polymer, silicon, and glass, none of which are particularly common irritants (Nguyen and Park 2018). However, irritation following a procedure may result from the MNs themselves or any substance used in conjunction.

Cercal Fucci-da-Costa and Reich Camasmie (2018) document a case of likely ICD due to skin rejuvenation MN therapy over the patient's dorsal hands with 10 applications with a $0.5 \mathrm{~mm}$ derma-roller. Following her MN therapy, the patient inadvertently applied arnica-based cream and developed yellowish papules compatible with $\mathrm{MN}$ perforation sites on an erythematous base $48 \mathrm{~h}$ post-arnica cream application. The authors attributed the lesions to ICD from the arnica-based cream due to the sparing of MN treated areas in which the cream was not applied and the patient's improvement $72 \mathrm{~h}$ post-topical corticosteroid treatment.

Assessment of post-procedural erythema allows a rough estimate of the irritation incurred during the procedure; however, the degree of post-procedural erythema is likely dependent on factors such as: MN application site, amount of MN applications, $\mathrm{MN}$ length and type, combination therapy with topical products, and variability between skin types.

Bal et al. (2008) applied MN arrays (200, 300, or $400 \mu \mathrm{m}$ solid metal MN arrays and 300 or $550 \mu \mathrm{m}$ hollow metal MN arrays) using a standardized electrical applicator to the forearms of 18 human volunteers and measured redness using skin color assessment and laser doppler imaging. They found longer needle lengths to result in greater irritation, evidenced by the $400 \mu \mathrm{m}$ solid MNs resulting in significantly greater change in redness in comparison to the $200 \mu \mathrm{m}$ solid MNs $(P<0.001)$. Lastly, they concluded $15 \mathrm{~min}$ post-application as the maximum change in redness with minimal irritation lasting less than $2 \mathrm{~h}$ for all MNs.

Gill et al. (2008) investigated safety of single, longer MN lengths $(480,700,960$, and $1450 \mu \mathrm{m})$ on the volar forearm of healthy human volunteers, finding decreasing erythema over $2 \mathrm{~h}$ in all subjects with no excessive erythema self-reported by research subjects when contacted at $24 \mathrm{~h}$.

Han Tae et al. (2012) used a chromameter to measure posttreatment erythema following MN therapy using 150 and $250 \mu \mathrm{m} \mathrm{MN}$ rollers over one side of the face of healthy human volunteers. They found recovery time to baseline erythema to be $24 \mathrm{~h}$ in the 5 -application group and $48 \mathrm{~h}$ in the 10 application group and a significant difference in the erythema 
index ratio between the two groups after $24 \mathrm{~h}(p=0.002)$. In contrast, they did not find a significant difference between the erythema index ratio of the 150 and $200 \mu \mathrm{m}$ MN roller groups, although the mean erythema index ratios were higher in the $250 \mu \mathrm{m} \mathrm{MN}$ roller group.

\subsection{Allergic contact dermatitis}

Allergic contact dermatitis (ACD) is a type IV hypersensitivity reaction requiring prior sensitization and re-exposure to the allergen (Mowad et al. 2016). In the sensitization phase, the unprocessed chemical allergen, known as a hapten, penetrates the lower levels of the epidermis, where it is engulfed by a Langerhans cell and later presented to T-cells (Marks and deLeo 2016). Upon subsequent exposure to the allergen, cell-mediated immune response results in an eczematous like lesion. As previously mentioned, microneedling disrupts the stratum corneum, increasing the ability of molecules, possibly allergens, to enter the dermis.

Yadav and Dogra (2016) identified a potential case of ACD occurring as a result of a $1.5 \mathrm{~mm}$ titanium-coated, stainless steel microneedling device for the treatment of atrophic acne facial scars. Other than a local anesthetic applied $1 \mathrm{~h}$ before and completely cleaned with normal saline and betadine, no serum or chemical was applied before, during or after the procedure. The patient developed erythema and edema over the next two days, which gradually subsided. Simultaneously, the patient developed vesiculopustular lesions and erythematous papules arranged linearly along the lines of the microneedling device, giving a "rail track appearance". The lesions cleared in 4 weeks with 5 days of $30 \mathrm{mg}$ oral prednisone and followed by mild topical corticosteroids, and after 3 months, she was patch tested with both nickel sulfate (5\% in petroleum) and titanium (10\% in petroleum) with readings taken at 48 and $96 \mathrm{~h}$. The patient demonstrated a negative reaction to titanium patch testing and tiny vesiculopustular lesions and intense erythema, extending beyond the margins at $48 \mathrm{~h}$ in response to nickel.

Of note Pahwa et al. (2012) and Dogra et al. (2014) document cases of "tram tracking" and "tram trek" scarring respectively. It is unclear whether Yadav and Dogra's documentation of a "rail track" appearing reaction represents a similar or distinct entity. However, the former authors were unable to attribute the scarring to a particular allergen or irritant, so these cases are discussed in the section, "irregular scarring".

\subsection{Allergic/irritant chronic inflammatory reactions and granulomas}

Granulomatous inflammation is most commonly characterized by a collection of histiocytes (macrophages) surrounding an antigenic center, which may occasionally be necrotic; however, granulomatous inflammation encompasses presentations ranging from a well organized granuloma to loose aggregates of epithelioid cells mixed with other inflammatory cells (Shah et al. 2017). Granulomas may develop with or without immunologic modulation in the case of granulomatous hypersensitivity and foreign-body granulomas respectively (Epstein 1989). Dermal granulomatous hypersensitivity has historically been associated with intradermal tattooing of red dyes with metallic elements and injection of dermal fillers such as hyaluronic acid and poly-L-lactic acid (PLA).

Pratsou and Gach (2013) report two females who underwent a facial microneedling procedure with a trained practitioner using a CE marked, FDA registered device (192 stainless steel MNs $1.5 \mathrm{~mm}$ long and $0.25 \mathrm{~mm}$ wide) coupled with skin cleansing and topical anesthetic cream. Within $24 \mathrm{~h}$, both sisters developed significant lymphadenopathy, and the older sister developed pinpoint erythema, malaise, and headache. Systemic antibiotics were unhelpful, as the older sister's condition worsened with a "florid erythematous papular rash over her face" with spread to her trunk and limbs. The patient gradually improved over two weeks with systemic and topical corticosteroid treatment. Biopsy of lesions showed a non-specific, chronic inflammatory infiltrate. Patch testing yielded a positive reaction to nickel sulphate (D4++), which was a known allergy to the patient. The authors were unable to attribute the reaction to her allergy, as the $\mathrm{MN}$ device contained up to $0.006 \%$ sulphur and $8 \%$ nickel bound to surgical grade stainless steel (per manufacturer)an amount thought to pose little or no risk in short term contact with nickel-sensitive individuals.

Soltani-Arabshahi et al. (2014) document a total of 3 cases of granulomatous reactions to MN therapy. The first two women presented after Dermapen MN therapy followed by high dose of lipophilic vitamin C (Vita C Serum; Sanítas Skincare) applied to the skin at the same medical spa. The Dermapen fraction microneedling device (Dermapen, LLC) is estimated to penetrate the skin anywhere from 0.25 $2.00 \mathrm{~mm}$. Both patients developed a progressive erythematous rash over the face in addition to systemic reactions, including arthralgias of varying intensity. In both cases, biopsy of indurated papules showed foreign body-type granulomatous reaction with focal, polarizable material present in giant cell cytoplasm. Patch testing both patients showed +1 reaction to Vita C Serum, while patch testing with Vita C Serum in 5 healthy volunteers yielded negative reactions. Both patients had persistent, mildly indurated, erythematous papules and plaques at 9-month follow-up. The last of three patients presented following three microneedling procedures, two in which a gel product (Boske Hydra-Boost Gel; Boske Dermaceuticals) and one in which Vital Pigment Stabilizer (Dermapen, LLC) were applied before microneedling. While the patient did not have systemic symptoms, she did present with a progressively worsening erythematous rash that developed papular features with a biopsy showing a similar granulomatous reaction. The 
last patient refused patch testing and demonstrated resolution at 3 weeks.

In order to limit chances of granuloma formation, patients should use only those products that are approved for intradermal use and prescribed by the physician in the immediate post-procedural time period. Alster and Graham (2018) typically allow patients to resume makeup application 2 days post-procedure and active skin care products 5 to 7 days after therapy.

\subsection{Irregular scarring}

Pahwa et al. (2012) reported "tram tracking" or multiple discrete papular scars in a linear pattern in the horizontal and vertical directions in a 25 year-old woman 1 month after treatment with a dermal rolling device (192 needles, $2 \mathrm{~mm}$ long) for management of post acne scarring. The scarring was predominantly located over the temporal area, zygomatic arch, and the forehead of the patient, who slightly improved with topical silicone gel at 6-month follow-up. They were unable to attribute the atypical scarring to an allergen or irritant.

Dogra et al. (2014) reported 2/36 patients undergoing $\mathrm{MN}$ therapy using a dermaroller (192 fine Microneedles, $1.5 \mathrm{~mm}$ in length and $0.1 \mathrm{~mm}$ in diameter) for atrophic acne scars to have a similar "tram trek" adverse effect. One patient developed severe tram-trek scarring over the malar prominence and was forced to withdraw; however, the patient did improve with topical tretinoin. The second patient developed less severe tram trek scarring over the forehead and was able to complete the study.

It is possible that the "tram trek" scarring may be a result of excessive force during the microneedling procedure over bony prominences of the patient's faces.

\subsection{Post-inflammatory hyperpigmentation}

Post-inflammatory hyperpigmentation (PIH) is a hypermelanosis resulting from dermal inflammation or injury most commonly in people with skin of color (Davis and Callender 2010). The inflammation may be endogenous in the case of primary dermatoses or exogenous from external insults like trauma or physical injury (Epstein 1989). While the pathogenesis is not completely known, PIH is believed to result from increased production of melanin or increased release of melanin due to melanocyte-stimulating signals such as cytokines and various other inflammatory mediators (Davis and Callender 2010).

Dogra et al. (2014) found 5/36 patients to present with post-inflammatory hyperpigmentation following 2-3 treatments of $\mathrm{MN}$ therapy for post-acne scarring. Of the five patients, three had severe hyperpigmentation forcing them to withdraw from the study, while the other two gradually improved with photoprotection.

Sharad (2011) and Majid (2009) reported more transient post-inflammatory hyperpigmentation in 4/36 patients and $1 / 37$ patients respectively in similar studies on MN therapy using a dermaroller (192 fine Microneedles, $1.5 \mathrm{~mm}$ in length and $0.25 \mathrm{~mm}$ in diameter) for atrophic scarring. Each of the previously mentioned cases of hyperpigmentation featured Fitzpatrick Type III or greater skin, except for one patient of unspecified type. To date, the amount of adverse hyperpigmentation reactions remain minimal and are possibly a result of improper UV protection following the procedure (Ramaut et al. 2018).

As in other skin resurfacing skin techniques, microneedling post-inflammatory hyperpigmentation is seen more commonly in skin of color. However, it should be noted the risk of hyperpigmentation is thought to be lower when compared to other dermabraisive techniques such as chemical peels and lasers (Cohen and Elbuluk 2016). We strongly recommend the use of test spots, post-procedural use of at least SPF 30 sunscreen, and avoidance of UV exposure both prior and after MN therapy to minimize chance of post therapy dyspigmentation. Please see Cohen and Elbuluk 2016 for an in depth review of uses and efficacy of MNs in skin of color.

\subsection{Other adverse effects to consider}

Lastly, other reactions of which MN users should be aware include photoirritation, photoallergy, Non-Immunologic Contact Urticaria (NICU), and Immunologic Contact Urticaria (ICU).

Phototoxic, or photoirritant, reactions are evoked from the combination of an exogenous or endogenous compound and ultraviolet (UV) irradiation (Ibbotson 2014). Phototoxic reactions most often appear similar to an acute sunburn; however, they occasionally present with urticarial, eczematous, lichenoid, or, rarely, pigmentary changes (Maibach and Honari 2014). In contrast, photoallergy requires a prior sensitization to the chemical in the presence of UV radiation and an additional exposure, in which there is a cell-mediated immune response and clinical presentation of the photoallergy (Ibbotson 2014). Photoallergic reactions most often present with eczematous like changes (Maibach and Honari 2014).

Contact urticaria comprises a group of inflammatory reactions ranging from itching, burning, and tingling to systemic anaphylaxis (Gimenez-Arnau and Maibach 2014). Contact urticaria can be divided into NICU and ICU, with the two separate entities distinguished by immunologic memory in the case of ICU, but no required prior sensitization in NICU (Gimenez-Arnau and Maibach 2014). ICU and NICU are distinct in mechanism and etiology, but have very subtle, if any, difference in their clinical presentation. (Gimenez-Arnau and Maibach 2014; Lahti 2000). 
There is little to no current literature documenting photoirritation, photoallergy, NICU, or ICU resulting from MN therapy; however, we believe they are important conditions of which to be aware with the potential growth of MN use in the future.

\section{Conclusion}

Of all $\mathrm{MN}$ applications, the growth of $\mathrm{MN}$ use in the cosmetics industry for skin rejuvenation in the spa or the home setting raises the most concern. As previously mentioned, skin rejuvenation with MNs or collagen-induction therapy represents the most extensive use of MNs in any one therapy of all potential $\mathrm{MN}$ applications. In addition, skin rejuvenation is frequently performed outside of the medical setting, which also increases the chance of adverse events, especially those stemming from improper sterilization or the coupling inappropriate topical products. We advise patients understand contraindications for use and follow post-therapy guidelines, especially when seeking therapy outside of the medical setting.

Ramaut et al. (2018) systematically reviewed the MN literature and its potential application in atrophic acne scars, skin rejuvenation, hypertrophic scars, keloids, striae distensae, androgenetic alopecia, melasma, and acne vulgaris. They generally favored MN therapy due to minimal side effects with shorter recovery periods and similar results when compared to other treatments.

We believe MNs may be a relatively safe alternative and, in some circumstances, a superior option to many conventional therapies. However, further research and experience is needed to clarify the most appropriate and safe way to utilize this technology.

\section{Compliance with ethical standards}

Conflict of interest The authors declare no conflict of interest.

\section{References}

T.S. Alster, P.M. Graham, Microneedling: A review and practical guide. Dermatol. Surg. 44(3), 397-404 (2018). https://doi.org/10.1097/dss. 0000000000001248

M.C. Aust, D. Fernandes, P. Kolokythas, H.M. Kaplan, P.M. Vogt, Percutaneous collagen induction therapy: An alternative treatment for scars, wrinkles, and skin laxity. Plast. Reconstr. Surg. 121(4), 1421-1429 (2008). https://doi.org/10.1097/01.prs.0000304612. 72899.02

M.M. Badran, J. Kuntsche, A. Fahr, Skin penetration enhancement by a microneedle device (Dermaroller()) in vitro: Dependency on needle size and applied formulation. Eur. J. Pharm. Sci. 36(4), 511-523 (2009). https://doi.org/10.1016/j.ejps.2008.12.008
S.M. Bal, J. Caussin, S. Pavel, J.A. Bouwstra, In vivo assessment of safety of microneedle arrays in human skin. Eur. J. Pharm. Sci. 35(3), 193-202 (2008). https://doi.org/10.1016/j.ejps.2008.06.016

S.M. Bal, A.C. Kruithof, H. Liebl, et al., In vivo visualization of microneedle conduits in human skin using laser scanning microscopy. Laser Phys. Lett. 7(3), 242-246 (2010) 22

A.P. Cercal Fucci-da-Costa, H. Reich Camasmie, Drug delivery after microneedling: Report of an adverse reaction. Dermatol. Surg. 44(4), 593-594 (2018). https://doi.org/10.1097/dss. 0000000000001250

S.I. Cho, B.Y. Chung, M.G. Choi, J.H. Baek, H.J. Cho, C.W. Park, et al., Evaluation of the Clinical Efficacy of Fractional Radiofrequency Microneedle Treatment in Acne Scars and Large Facial Pores. Dermatol. Surg 38(7pt1), 1017-1024 (2012). https://doi.org/10. $1111 / \mathrm{j} .1524-4725.2012 .02402 . x$

B.E. Cohen, N. Elbuluk, Microneedling in skin of color: A review of uses and efficacy. J. Am. Acad. Dermatol. 74(2), 348-355 (2016). https:// doi.org/10.1016/j.jaad.2015.09.024

N.M.M.D. Cunha, S.L.A. Campos, A.I.P.C. Fidalgo, Unusual presentation of tinea Corporis associated with the use of a microneedling device. Aesthet. Surg. J. 37(7), NP69-NP72 (2017). https://doi.org/ 10.1093/asj/sjx086

E.C. Davis, V.D. Callender, Postinflammatory hyperpigmentation: A review of the epidemiology, clinical features, and treatment options in skin of color. J. Clin. Aesthet. Dermatol. 3(7), 20-31 (2010)

R. Dhurat, S. Mathapati, Response to microneedling treatment in men with androgenetic alopecia who failed to respond to conventional therapy. Indian. J. Dermatol. 60(3), 260-263 (2015). https://doi.org/ 10.4103/0019-5154.156361

R. Dhurat, M. Sukesh, G. Avhad, A. Dandale, A. Pal, P. Pund, A randomized evaluator blinded study of effect of microneedling in androgenetic alopecia: A pilot study. Int. J. Trichology. 5(1), 6-11 (2013). https://doi.org/10.4103/0974-7753.114700

S. Dogra, S. Yadav, R. Sarangal, Microneedling for acne scars in Asian skin type: an effective low cost treatment modality. J. Cosmet. Dermatol 13(3), 180-187 (2014). https://doi.org/10.1111/jocd. 12095

R.F. Donnelly, T.R.R. Singh, M.M. Tunney, D.I.J. Morrow, P.A. McCarron, C. O'Mahony, et al., Microneedle arrays allow lower microbial penetration than hypodermic needles in vitro. Pharm. Res. 26(11), 2513-2522 (2009). https://doi.org/10.1007/s11095009-9967-2

L. Engelke, G. Winter, S. Hook, J. Engert, Recent insights into cutaneous immunization: How to vaccinate via the skin. Vaccine 33(37), 4663-4674 (2015). https://doi.org/10.1016/j.vaccine.2015.05.012

J.H. Epstein, Postinflammatory hyperpigmentation. Clin. Dermatol. 7(2), 55-65 (1989). https://doi.org/10.1016/0738-081X(89)90057-6

G. Fabbrocini, N. Fardella, A. Monfrecola, I. Proietti, D. Innocenzi, Acne scarring treatment using skin needling. Clin. Exp. Dermatol 34(8), 874-879 (2009). https://doi.org/10.1111/j.1365-2230.2009.03291.x

D. Fernandes, Minimally invasive percutaneous collagen induction. Oral. Maxillofac. Surg. Clin. 17(1), 51-63 (2005).

H.S. Gill, D.D. Denson, B.A. Burris, M.R. Prausnitz, Effect of microneedle design on pain in human subjects. Clin. J. Pain 24(7), 585-594 (2008). https://doi.org/10.1097/AJP.0b013e31816778f9

Gimenez-Arnau AM, Maibach HI. Contact Urticaria Syndrome. CRC Press; (2014)

J. Gupta, H.S. Gill, S.N. Andrews, M.R. Prausnitz, Kinetics of skin resealing after insertion of microneedles in human subjects. J. Control. Release 154(2), 148-155 (2011). https://doi.org/10.1016/ j.jconrel.2011.05.021

Y. Han Tae, Y. Park Kui, Y. Ahn Ji, W. Kim Seo, J. Jung Hye, B.J. Kim, Facial Skin Barrier Function Recovery After Microneedle Transdermal Delivery Treatment. Dermatol. Surg. 38(11), 18161822 (2012). https://doi.org/10.1111/j.1524-4725.2012.02550.x 
M.I. Haq, E. Smith, D.N. John, et al., Clinical administration of microneedles: skin puncture, pain and sensation. Biomed. Microdevices 11, 35-47 (2009) 23

S.H. Ibbotson, in Applied Dermatotoxicology, ed. by G. Honari. Chapter 5 - Photoallergic contact dermatitis: Clinical aspects A2 Maibach, Howard (Academic Press, Boston, 2014), pp. 85-114

J. Jiang, J.S. Moore, H.F. Edelhauser, M.R. Prausnitz, Intrascleral drug delivery to the eye using hollow microneedles. Pharm. Res. 26(2), 395-403 (2009). https://doi.org/10.1007/s11095-008-9756-3

J.C. Joyce, T.D. Carroll, M.L. Collins, C. M-h, L. Fritts, J.C. Dutra, et al., A microneedle patch for measles and rubella vaccination is immunogenic and protective in infant rhesus macaques. J. Infect. Dis. 218(1), 124-132 (2018). https://doi.org/10.1093/infdis/jiy139

M.N. Kelchen, K.J. Siefers, C.C. Converse, et al., Micropore closure kinetics are delayed following microneedle insertion in elderly subjects. J. Control. Release 225, 294-300 (2016)

R.T. Kenney, S.A. Frech, L.R. Muenz, C.P. Villar, G.M. Glenn, Dose sparing with intradermal injection of influenza vaccine. N. Engl. J. Med. 351(22), 2295-2301 (2004). https://doi.org/10.1056/ NEJMoa043540

T. Kiang, S. Ranamukhaarachchi, M. Ensom, Revolutionizing therapeutic drug monitoring with the use of interstitial fluid and microneedles technology. Pharmaceutics 9(4), 43 (2017)

S.E. Kim, J.H. Lee, H.B. Kwon, B.J. Ahn, A.Y. Lee, Greater Collagen Deposition with the Microneedle Therapy System Than with Intense Pulsed Light. Dermatol. Surg 37(3), 336-341 (2011). https://doi. org/10.1111/j.1524-4725.2011.01882.x

A. Lahti, in Handbook of Occupational Dermatology, ed. by L. Kanerva, J. E. Wahlberg, P. Elsner, H. I. Maibach. Non-Immunologic Contact Urticaria (Springer Berlin Heidelberg, Berlin, 2000), pp. 221-224

H. Leatham, L. Guan, A.L.S. Chang, Unintended widespread facial autoinoculation of varicella by home microneedling roller device. JAAD Case Reports 4(6), 546-547 (2018). https://doi.org/10.1016/ j.jdcr.2018.02.004

I. Leroux-Roels, E. Vets, R. Freese, M. Seiberling, F. Weber, C. Salamand, et al., Seasonal influenza vaccine delivered by intradermal microinjection: A randomised controlled safety and immunogenicity trial in adults. Vaccine 26(51), 6614-6619 (2008). https://doi. org/10.1016/j.vaccine.2008.09.078

C.G. Li, C.Y. Lee, K. Lee, H. Jung, An optimized hollow microneedle for minimally invasive blood extraction. Biomed. Microdevices 15(1), 17-25 (2013). https://doi.org/10.1007/s10544-012-9683-2

G. Ma, C. Wu, Microneedle, bio-microneedle and bio-inspired microneedle: A review. J. Control. Release 251, 11-23 (2017). https://doi.org/10.1016/j.jconrel.2017.02.011

H. Maibach, G. Honari, Chapter 3 - Photoirritation (Phototoxicity): Clinical Aspects. Applied Dermatotoxicology (Academic Press, Boston, 2014), pp. 41-56

I. Majid, Microneedling therapy in atrophic facial scars: An objective assessment. J Cutan Aesthet Surg 2(1), 26-30 (2009). https://doi. org/10.4103/0974-2077.53096

J.G. Marks, V.A. DeLeo, Contact \& Occupational Dermatology (Medical Publishers Pvt. Limited, Jaypee Brothers, 2016)

C.M. Mowad, B. Anderson, P. Scheinman, S. Pootongkam, S. Nedorost, B. Brod, Allergic contact dermatitis: Patient management and education. J. Am. Acad. Dermatol. 74(6), 1043-1054 (2016). https:// doi.org/10.1016/j.jaad.2015.02.1144

T.T. Nguyen, J.H. Park, Human studies with microneedles for evaluation of their efficacy and safety. Expert. Opin. Drug. Deliv. 15(3), 235-45 (2018).
M. Pahwa, P. Pahwa, A. Zaheer, "Tram track effect" after treatment of acne scars using a microneedling device. Dermatol. Surg. 38(7), 1107-1108 (2012). https://doi.org/10.1111/j.1524-4725. 2012.02441.x

P. Pratsou, J. Gach, Severe systemic reaction associated with skin microneedling therapy in 2 sisters: A previously unrecognized potential for complications? J. Am. Acad. Dermatol. 68(4), $6447(2013)$

L. Ramaut, H. Hoeksema, A. Pirayesh, F. Stillaert, S. Monstrey, Microneedling: Where do we stand now? A systematic review of the literature. J. Plast. Reconstr. Aesthet. Surg. 71(1), 1-14 (2018). https://doi.org/10.1016/j.bjps.2017.06.006

N.G. Rouphael, M. Paine, R. Mosley, S. Henry, D.V. McAllister, H. Kalluri, et al., The safety, immunogenicity, and acceptability of inactivated influenza vaccine delivered by microneedle patch (TIV-MNP 2015): A randomised, partly blinded, placebo-controlled, phase 1 trial. Lancet 390(10095), 649-658 (2017). https:// doi.org/10.1016/S0140-6736(17)30575-5

K.K. Shah, B.S. Pritt, M.P. Alexander, Histopathologic review of granulomatous inflammation. J. Clin. Tuberc. Other. Mycobact. Dis. 7, 112 (2017). https://doi.org/10.1016/j.jctube.2017.02.001

J. Sharad, Combination of microneedling and glycolic acid peels for the treatment of acne scars in dark skin. J. Cosmet. Dermatol 10(4), 317-323 (2011). https://doi.org/10.1111/j.1473-2165.2011.00583.x

A. Singh, S. Yadav, Microneedling: Advances and widening horizons. Indian. J. Dermatol. 7(4), 244-254 (2016). https://doi.org/10.4103/ 2229-5178.185468

R. Soltani-Arabshahi, J.W. Wong, K.L. Duffy, D.L. Powell, Facial allergic granulomatous reaction and systemic hypersensitivity associated with microneedle therapy for skin rejuvenation. JAMA. Dermatol. 150(1), 68-72 (2014). https://doi.org/10.1001/jamadermatol.2013. 6955

C.-H. Tan, S. Rasool, G.A. Johnston, Contact dermatitis: Allergic and irritant. Clin. Dermatol. 32(1), 116-124 (2014). https://doi.org/10. 1016/j.clindermatol.2013.05.033

L. Torezan, Y. Chaves, A. Niwa, J.A.J. Sanches, C. Festa-Neto, R.-M. Szeimies, A pilot Split-face study comparing conventional methyl Aminolevulinate-photodynamic therapy (PDT) with microneedlingassisted PDT on actinically damaged skin. Dermatol. Surg. 39(8), 1197-1201 (2013). https://doi.org/10.1111/dsu.12233

P. Van Damme, F. Oosterhuis-Kafeja, M. Van der Wielen, Y. Almagor, O. Sharon, Y. Levin, Safety and efficacy of a novel microneedle device for dose sparing intradermal influenza vaccination in healthy adults. Vaccine 27(3), 454-459 (2009). https://doi.org/10.1016/j.vaccine. 2008.10.077

E.M. Vicente-Perez, E. Larrañeta, M.T.C. McCrudden, A. Kissenpfennig, S. Hegarty, H.O. McCarthy, et al., Repeat application of microneedles does not alter skin appearance or barrier function and causes no measurable disturbance of serum biomarkers of infection, inflammation or immunity in mice in vivo. Eur. J. Pharm. Biopharm. 117, 400-407 (2017). https://doi.org/10.1016/j.ejpb. 2017.04.029

S. Yadav, S. Dogra, A cutaneous reaction to microneedling for Postacne scarring caused by nickel hypersensitivity. Aesthet. Surg. J. 36(4), NP168-NNP70 (2016). https://doi.org/10.1093/asj/sjv229

Publisher's note Springer Nature remains neutral with regard to jurisdictional claims in published maps and institutional affiliations. 